\title{
Technical skills assessment in thoracic surgery education: We won't get fooled again
}

\author{
Marc R. Moon, MD
}

See related article on pages 2491-6.

Dr Irv Kron's American Association for Thoracic Surgery Presidential Address in 2011 was a lightbulb moment for me. "No resident should complete a program incompetent to perform surgery," he challenged, "The responsibility is on us." Dr Kron encouraged us to refocus how we teach technical surgery and to define technical standards-but how? The TSDA has done an outstanding job in defining the specific knowledge base that a thoracic surgeon should possess, and its assessment is relatively straightforward. The written boards do an outstanding job of assessing a candidate's fund of knowledge, and the oral boards can identify a satisfactory comprehension of the flow of an operation and intraoperative decision making. It is the evaluation of technical capabilities that has historically been limited to a subjective assessment, an assessment that may be challenging in current times given the lack of opportunity for thoracic surgery residents to perform critical tasks truly independent of an attending's constant supervision. In the mid to late 1900 s, the "ward service" afforded the opportunity for residents to operate independently. Those days are gone, to the benefit of our patients, but the downside is that we no longer have the opportunity to evaluate the truly independent technical competence of our trainees. In the operating room, we continually evaluate and correct needle angles, graft orientation, bite technique, and spacing on every anastomosis a resident performs. There is no room for trial and error during training, only what I refer to as "continually supervised, corrected perfection." Dr John Alexander initiated the first training program in thoracic surgery at the University of Michigan in 1928. He proclaimed, "The time has passed when surgeons must gain their experience at the expense of their patients." ${ }^{2}$ He thought that after 2 years of intensive study, trainees in thoracic surgery would be prepared for independent practice, but is this truly the case in 2014 ? We have to make certain to not simply transfer Dr Alexander's

\footnotetext{
From the Division of Cardiothoracic Surgery, Washington University School of Medicine, St Louis, Mo.

Disclosures: Author has nothing to disclose with regard to commercial support.

Received for publication Sept 18, 2014; accepted for publication Sept 19, 2014; available ahead of print Oct 16, 2014.

Address for reprints: Marc R. Moon, MD, Division of Cardiothoracic Surgery, Washington University School of Medicine, 660 S. Euclid Ave, Box 8234, St

Louis, MO 63110-1013 (E-mail: moonm@wustl.edu).

J Thorac Cardiovasc Surg 2014;148:2497-8

0022-5223/\$36.00

Copyright (c) 2014 by The American Association for Thoracic Surgery

http://dx.doi.org/10.1016/j.jtcvs.2014.09.057
}

"expense" from the period of training to the early years of practice.

Simulators, as developed by the TSDA and Joint Council on Thoracic Surgery Education, permit trial by error and unsupervised practice, and likely contribute to the resident's "10,000 hours" toward excellence. ${ }^{3}$ The American Board of Thoracic Surgery duly has included simulation time as a requirement to sit for the Boards. The next logical step would be to develop a technical assessment tool that could be used to evaluate the capabilities of American Board of Thoracic Surgery candidates, but studies are essential to validate assessment tools if they are to be used for passand fail-type decisions during residency training or board certification. In 2013, Lee and coauthors ${ }^{4}$ demonstrated high inter-rater reliability and internal consistency reliability for the coronary anastomosis assessment tool created by the Joint Council. The evaluators received no specific training in this original study. It was assumed that the consistency among raters was due to the experience of the surgeons whose years on staff ranged from 2 to 33 , with a mean of 13.4 years. In the current study by Lou and associates, ${ }^{5}$ inter-rater reliability ranged from poor to moderate at baseline but improved to moderate to strong after a 30-minute training session for the evaluators. The initial suboptimal inter-rater reliability results were attributed to the evaluators being less experienced; the mean years on staff was 4.5 , and only $33 \%$ were on staff more than 5 years. It is my contention that rather than years on faculty being the important difference in these studies, it was the specialty of the evaluators that led to the disparate results. In the original study by Lee and colleagues, ${ }^{4} 8$ of 10 attending surgeons specialized in cardiac surgery and thus likely had experience evaluating coronary anastomoses intraoperatively, including bite, needle holder use, needle angles, and hand mechanics. In the follow-up study by Lou and colleagues, ${ }^{5}$ only 2 of 9 attendings were cardiac surgeons, and the other 7 identified as general thoracic surgeons may not have been as familiar with the clinical nuances of coronary anastomoses. Regardless of the reason for the difference between the 2 reports, the important finding is that surgeons who are not necessarily experienced in technical assessment can be taught to evaluate coronary anastomoses consistently and reproducibly after a short period of proper training. Studies such as these are critical to standardize the evaluation of surgical technique, shifting the subjective to the objective during assessment.

The findings of Lou and colleagues ${ }^{5}$ reinforce the Educate the Educators concept that although teaching and 
evaluation are learned traits, traits we all believe we acquire during residency and beyond, the evaluation of our educational efforts can benefit from standardization. Gone, I hope, are the days of the Board Examiner creating his own questions on the fly or the days of our assessment of surgical preparedness merely on the vibe we feel following a case with a trainee whose demeanor was pleasant but whose surgical skills we may not have adequately assessed. Standardization is critical not only to ensure a satisfactory product on completion of training but also to ensure fairness in evaluation to the examinee whose efforts to prepare for a career in thoracic surgery need not rest on the whim of an examiner who may be having a "bad day." The effort of the TSDA and Joint Council to develop an objective evaluation tool for assessment of technical skill is to be applauded. Program directors need an objective mechanism to evaluate the adequacy of technical skills. Our field is highly technical, and the assessment of the technical prowess of our trainees needs to match in severity the potential consequences of a trainee's release who may not yet be ready for prime time. Identification of deficiencies early can prompt counseling to focus on a field whose technical skill is commensurate with both their capabilities and interests.

Dr Kron noted graduating 40 residents without a single failure during his 20-year reign as program director at the University of Virginia. ${ }^{1}$ I have been Program Director at Washington University for 11 years, during which time 34 residents have completed our program. In most endeavors, 33 of 34 or a $97 \%$ rate of success would be considered outstanding, but in thoracic surgery education, anything less than $100 \%$ is on us as instructors and evaluators. This resident was outstanding in preoperative and postoperative care, as well as understanding the conduct of a complex operation, but the intricacies of a small coronary anastomosis performed without "continually supervised, corrected perfection" proved beyond the resident's capacity. It turns out the resident had an unrecognized visual limitation that did not manifest until independent unsupervised performance. I have often pondered since, how could we have identified this resident's limitation before completing our residency program? The transformation of a trainee into a successful thoracic surgeon relies on our capability to assess technical abilities. Sure, we all know a great surgeon when we see one, but the real trick is to be able to differentiate the competent surgeon when he or she has not yet had the opportunity to truly perform complex, intricate technically demanding skills independently. A competent surgeon does not necessarily need to move his hands fast—-speed is not one of the assessment variables described in the reports by Lee and colleagues ${ }^{4}$ or Lou and colleagues ${ }^{5}$-but having technical accuracy and efficiency in motion, staying organized, and not repeating steps are the key.

Reproducible measures of adequate technical skill beyond the subjective should become part of the Board's evaluation process at some point in the future. Simulation tools as described by Lou and associates ${ }^{5}$ make it possible to define surgical technical skills objectively. Simulation is now required by the Board and rightfully so, and although simulation does not replace the operating room environment, in our particular resident's case, it likely would have identified the critical technical flaw that we did not appreciate, the technical flaw that was not unmasked until a truly independent unsupervised performance. Thankfully, no patients suffered as a consequence and the deficiency was identified swiftly and corrective action taken, but to the surgeons who had to carry the burden of what we now realize was a subpar technical resident, I apologize. I am told by my wife that it may be an old Chinese proverb, but I first heard it from Dean Smith, the North Carolina Tar Heels' legendary basketball coach, during an interview years ago, "Fool me once, shame on you. Fool me twice, shame on me." Thanks to the tools being developed by the TSDA and Joint Council, to quote 2 other prophets from my youth, Roger Daltrey and Pete Townshend, I hope we "won't get fooled again!"

\section{References}

1. Kron IL. Surgical mentorship. J Thorac Cardiovasc Surg. 2011;142:489-92.

2. Alexander J. The training of a surgeon who expects to specialize in thoracic surgery. J Thorac Surg. 1936;5:579-82.

3. Gladwell M. Outliers. New York: Little Brown; 2008.

4. Lee R, Enter D, Lou X, Feins RH, Hicks GL, Gasparri M, et al. The Joint Council on Thoracic Surgery Education coronary artery assessment tool has high interrater reliability. Ann Thorac Surg. 2013;95:2064-70.

5. Lou X, Lee R, Feins RH, Enter D, Hicks GL, Verrier ED, et al. Training lessexperienced faculty improves reliability of skills assessment in cardiac surgery. J Thorac Cardiovasc Surg. 2014;148:2491-6. 